\title{
ARM BASED AUTOMATIC CONTROL SYSTEM OF NANO POSITIONING STAGE FOR MICROMANUFACTURING
}

\author{
Gaurav Singh Naruka ${ }^{1}$, Niharika Jha ${ }^{1}$ and Himanshu Dutt Sharma ${ }^{2}$ \\ ${ }^{1}$ International Institute of Information Technology(I2IT), \\ Pune-411057(Mah.)India \\ gauravnaruka3322@gmail.com, niharikajha2509@gmail.com \\ ${ }^{2}$ CSIR-Central Electronics Engineering Research Institute(CEERI), \\ Pilani-333031(Raj.)India \\ hdsharma@gmail.com, hosharma@ceeri.ernet.in
}

\begin{abstract}
A microcontroller based control system to drive the Physik Instrumente ${ }^{\circledR}\left(P I{ }^{\circledR}\right)$ piezoelectric ultrasonic nano-positioning (PUN) stage for a micro-factory has been proposed by the author. The tuning parameters of the PI ${ }^{\circledR}$ Line Controller are chosen such that the PUN stage shows optimum step response. The microcontroller i.e. LPC2478R provides the user with the choices of operations on the 3.2" QVGA LCD screen and the choice can be made by a 5-key joystick. The PUN stage moves in different geometrical patterns as chosen by the user. The stage is placed in the workspace of the Clark-MXRR,Inc. CPA-2101 femto-second laser. Different patterns are made on the material in question. As compared to the previous works in this area, the user is given the power for position control, real time tracking, and trajectory planning of the actuator. The user interface has been made very easy to comprehend. The repeatability of tasks, portability of the as- sembly, the reduction in the size of the system, power consumption and the human involvement are the major achievements after the inclusion of a microcontroller.
\end{abstract}

\section{INTRODUCTION}

As the dimensions of the mechanical parts of products are on the decline, manufactur- ing systems are becoming larger and more complicated. However the paradigm shift from the micro scale to the nano scale with the advent of VLSI circuits and devices, ensured that a change was brought about in the concept of a microfactory . Systems became more specific to material types, component geometries, and types of mechani- cal motions that can be realized and cost. The technique of MEMS eventually became slow, sequential and hence not suitable to mass production $[10,12]$.High speed and positional accuracy can also be achieved by using PUN actuators which have an excel- lent operating bandwidth, can generate large forces from a compact size and achieve a positional accuracy of under several nano-meters [1].The scope of using PUN actuators increases majorly once its control strategy has been devised. Most of the works in this area have dealt with the development of control approaches to perform tasks such as David C. Wyld (Eds) : ICCSEA, SPPR, CSIA, WimoA, SCAI - 2013

pp. 375-383, 2013. () CS \& IT-CSCP 2013

DOI : $10.5121 /$ csit.2013.3539 
positioning, orientation, picking, placing, and insertion of the different micro objects in grids or in between plates[13].

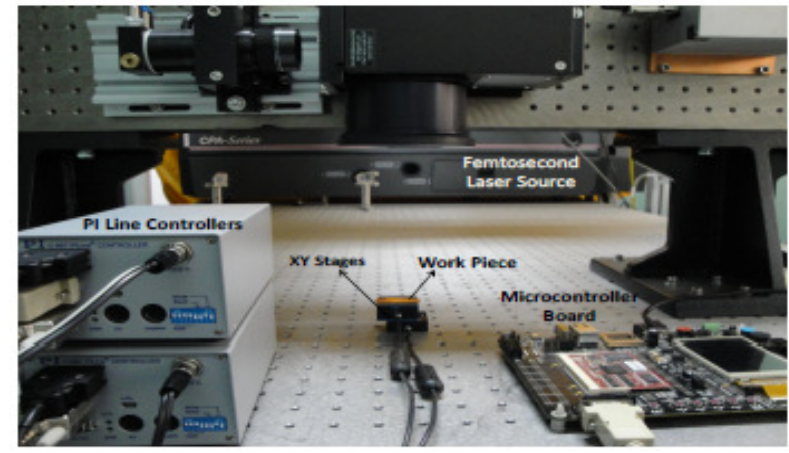

Figure 1: Femtosecond Laser Micromanufacturing Setup

Enabling a 2 DOF through a Piezo actuator can foray its use in Characterization of microdevices, Manufacturing and assembly of micro devices, High resolution industrial applications such as optical fiberalignment, biological cell manipulation, and scanning probe microscopy (SPM) $[2,3,4,13]$.

Authors propose the use of a 32 bit ARM microcontroller to establish a serial connection with the PILine Controller C867 which essentially uses the PID servo control to drive the PI stage M663. A joystick (interfaced with the microcontroller) is used to choose the controls displayed on an LCD screen (also interfaced to the same micro controller).Thus the system features the piezoelectric ultrasonic standing wave motor driven by a PILine Controller taking commands from the user through the microcontroller. Paper is divided into nine sections; Section 2 is the description of the hardware involved in the micromanufacturing workcell. Section 3 discusses the flow of signal to analyse the control elements of the system and explains graphically the conclusions of the experiments conducted by tuning the PID controller .Section 4 is the description how the communication between the components of the automatic control system was designed. Section 5 includes the process of realizing different geometries. Section 6 explains the execution of the tasks in the micromanufacturing system. Section 7 gives information about the future scope of the experiments conducted and the last section concludes the work.

\section{DESCRIPTION OF THE MICROMANUFACTURING WORKCELL}

The Fig. 1 shows the entire set up of the micromanufacturing system,employed by the author, at work .The Fig. 2 shows briefly the structure and the main blocks of this system .

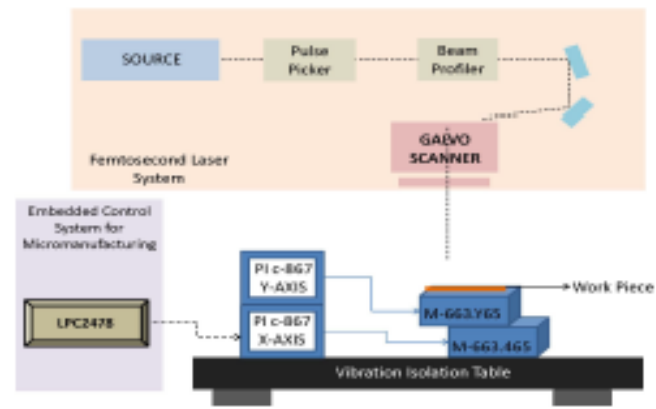

Figure 2: Block Daigram of the Micro manufacturing System

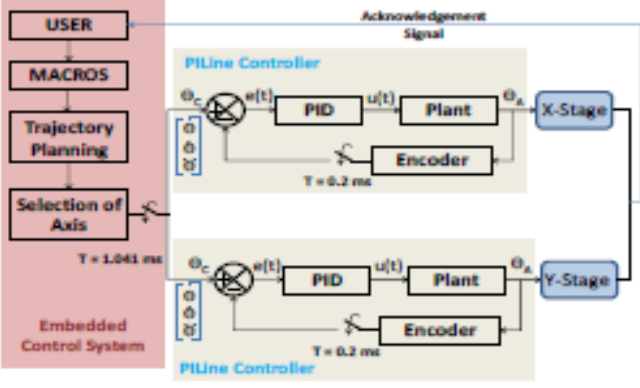

Figure 3: Control Architecture of the Ultrasonic Motor 


\subsection{LPC2478}

It is a $16 / 32$ bit RISC microcontroller that has $512 \mathrm{kB}$ of on-chip high-speed flash mem- ory. A development board named Embedded Artists' LPC2478-32 OEM Board v1.3 is used. The board includes: 3.2 inch QVGA TFT colour LCD with touch screen panel, RS232 male terminal, 5-key joystick, Ethernet connector (RJ45), MMC/SD interface \& connector, CAN interface \& connector and many other useful features.[5]

\subsection{PILine ${ }^{\circledR}$ Controller}

The C-867 controller is a compact case enclosing both, the driver electronics for the PUN motors and components for controlling and communication. The controller can be operated by a host PC either via a USB port or a RS-232 interface or manually by a joy-stick [6].Two controllers connected in daisy chain topology have been used to drive 2 Piezo-motors which enable a 2 DOF.

\subsection{PI Stage}

The advantages of using Piezo-actuators have already been discussed in the introduction. The PI M-663 is a Precision-class micropositioning stage with a non-contact optical linear encoder for measuring the distance travelled by the stage. The minimum incremental motion is 300 nanometer and sensor resolution is 100 nanometer. The maximum velocity it can obtain is $400 \mathrm{~mm} / \mathrm{sec}$. Its travel range is $19 \mathrm{~mm}$ [7]. Two PI stages have been placed on top of each other to ascertain a 2 DOF. The movement of each stage can be individually controlled.

\subsection{Femtosecond Laser}

Ultrashort laser pulses have ability for minimal damage and precise processing. Because of the melting effects around the machined site and the unacceptable degradation in the edge quality of the microstructure, the use of nanosecond pulses was discouraged and was replaced by femtosecond / picosecond lasers [8]. The TI: Sapphire CPA series femtosecond version of the laser by Clark-MXR, Inc was used. This assembly produces a laser pulse having energy of less than $.8 \mathrm{~mJ}$ at $1 \mathrm{kHz}$ and pulse width of less than 150fs. [9]

\section{CONTROL STRATEGY}

To devise the control strategy of this assembly, the signal flow in the system is traced and further used to determine or estimate the transfer functions of the controller and the plant.

\subsection{Working of the Plant}

The PUN actuator used here is entirely defined as a Standing Wave Ultrasonic Piezo-electric Linear Motor. The working of this rare class of motors can be briefly described as follows. Two orthogonal vibration components, when coupled, create a 2-D standing wave in the piezo-ceramic (PIC181) electrodes. [16], An alumina tip placed on top of these electrodes follows an elliptical trajectory, as a result of these vibrations, The aluminium plate which is in contact with the tip receives micro-impulses from it during the course of the tip's trajectory, which causes it to move forward or backward according to the angles which these impulses are given.[15] If seen in 
analogy with any motor, the peizoceramic electrodes play the role of the stator, where as the moving aluminium plate becomes the rotor. Researchers have generated mathematical models for this motor considering it as a spring-mass-damper system [14]. This trajectory is generally trapezoidal, meaning there is acceleration till the desired velocity is reached, the velocity remains constant till a certain position, and then there is a deceleration as controlled by the closed loop servo control.

\subsection{Study of Controller Dynamics}

There are traditional methods of extracting the plant dynamics, if the transfer function of the controller is estimated. However, few of these can be applied to the system in consideration due to the constraints in the type of input signal applied, adjustments in fundamental frequency, and non availability of certain piezo-electric constants necessary for mathematical modelling. The Proportional(P), Integral(I) and Derivative(D) constants are changed using the PI Tuning Tool in the PI Mikromove application. The graphs below are plotted using MATLAB. They show the time-domain response of the moving stage using three different sets of P, I, D terms. The Graph 4(a) shows the behaviour of the stage using P-10, I-15, D-15. Here the settling time was unacceptably long and there is a noticeable overshoot making the system under-damped. Graph 4(b) is plotted using P-30, I-100,D-90. It shows an overdamped system with damping factor greater than 1 . However there is a slight increase in the rise time of this time response.

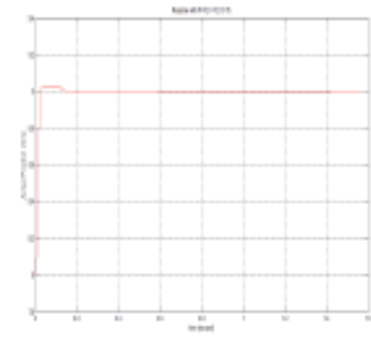

(a)

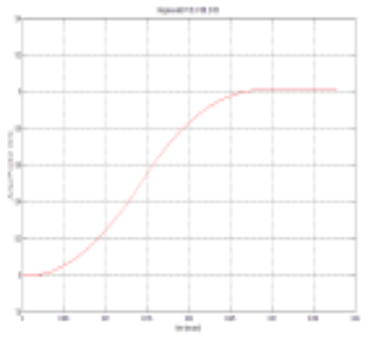

(b)

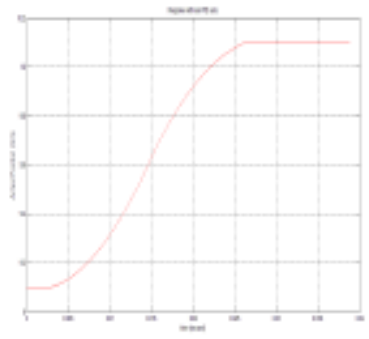

(c)

Figure 4: Responses of PID

Graph 4(c) is the behaviour of the stage using a combination of the sets mentioned above.The time domain response of this combination shows an overdamped system with a rise time better than in Fig. 4(b) and a settling time lesser than in Fig. 4(a).This implies that 2 or more than 2 P,I,D sets should be used for the actuator to ensure no vibrations and optimized rise time and settling time. The analysis of the Fig. 4(a) and (b) provides a solution for optimizing the rise time and the settling time. A combination of these 2 sets in a manner such that when the target position is relatively far the P,I,D values in the set 1 are used and when the target position close the P,I,D values in the set 2 are used.

\subsection{Control Architecture}

The signal flow from the microcontroller to the actuator is described in steps below and in Fig. 3. The user uses the joystick to make choices maximum velocity, acceleration and the geometry to be traced. The microcontroller converts these decisions into macros. It plans the trajectory of the coupled stages and selects the appropriate stage to be moved according to the geometry, The 
target position, maximum velocity and the maximum acceleration of each of the stages will be sent to their individual PILine controllers, According to the target position the stage is moved. The encoder obtains the actual position is obtained at $.2 \mathrm{~ms}$. The difference between the actual position and the target position is the error signal e $(\mathrm{t})$. This error signal is fed to the PID controller according to which the control signal $u(t)$ is generated, The control signal is a voltage in the range of -10 to $+10 \mathrm{~V}$ which is applied as an excitation voltage to the piezoceramic plates. This brings about the movement in the stage, When the geometry chosen is completely realised, an acknowledgement signal is generated and sent to the user. Thus the user will be able to continue with his next task.

\section{DESIGN OF ThE AUtOMATIC CONTROL SySTEM}

The Fig. 5 highlights the flow of data from the microcontroller to the actuator.

The automatic control system is an amalgamation of optimal tuning of the PI ${ }^{\circledR}$ Line controller and the transfer of commands through the microcontroller. The knowledge of UART is essential for this design. A few points were kept in mind while communicating through LPC2478. The PILine ${ }^{\circledR}$ Controller recognizes text based commands sent in a specific

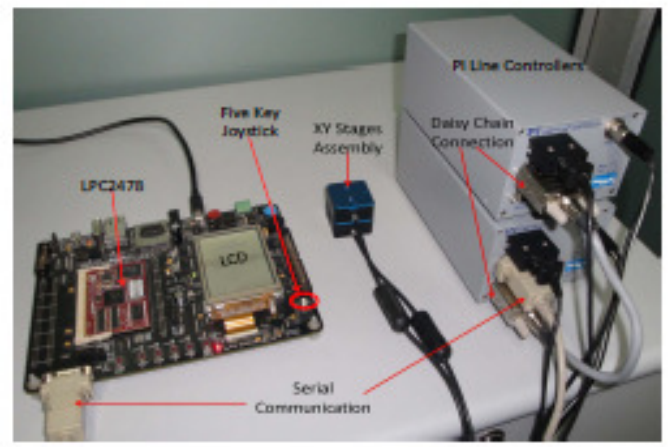

Figure 5: Setup for the Embedded Control System

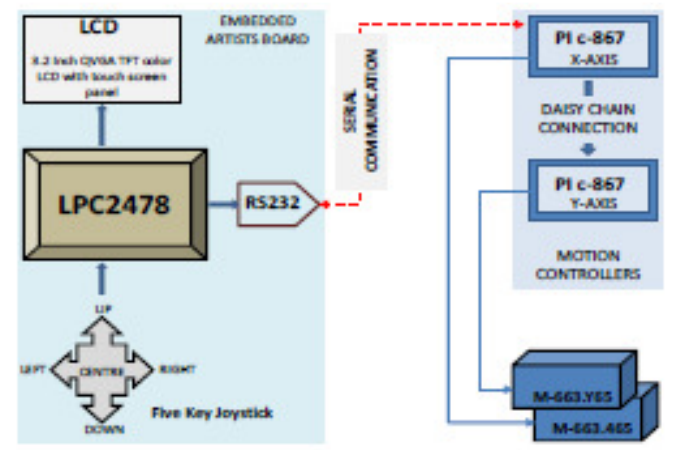

Figure 6: Embedded Control System

format as specified by the PI ${ }^{\circledR}$ General Command Set, One or more sets of these commands can be stored in a file and/or transmitted over RS-232 with the baud rate set the same as that of the PI ${ }^{\circledR}$ Line controller, Each controller in a daisy chain topology has a unique address. While in daisy chain topology the addresses of the target controller and the sender are required in every command line. [6], The number of data bits, parity bit and stop bit settings are also made considering mode switches in the front panel of the Line Controller (in our case 8 data bits, no parity and one stop bit), The ASCII character of the sequence " $\backslash \mathrm{n}$ " for a new line has to be sent after every command line.

\section{REALIZATION OF DIFFERENT GEOMETRIES}

The Fig. 7(a), 7(b), 7(c) and 7(d) display the basic algorithms adopted by the user interface routine to realize different geometrical patterns. As seen, the user can make the stages move in different geometries and set individual velocities for each task without switching the servo motor 
on/off, till he decides to exit. The pattern that will be made by the laser on the work piece is plotted roughly in the Fig. 8. This plotting is done using image processing. The red spot made by the laser beam was tracked when the 2 stages were in movement.

\subsection{Software Design of the User Interface}

The choice of realizing different geometrical patterns using the 5 position joystick is displayed on the LCD provided on the board. The current co-ordinates of the stage are also shown on the LCD and hence tracking of the stage is assured. Assigning the velocity $(\max 400 \mathrm{~mm} / \mathrm{sec})$ and the acceleration with which the stage is moving is also facilitated. Fig. 9 shows the different windows created for the user interface. Window (A) is the first screen informing the user that the XY stages have been initialized and the instruction for moving to the exit screen has been displayed at the bottom. Window (B) is the home screen where the user can make choices for diferent geometrical patterns and also choose the velocity at which the stages must move. Window (C) displays the tracking co-ordinates of the stages when the stages were moving in circular geometry. Window (D) shows the exit window after the user decides that he has executed all of his tasks. As is seen at every stage except for window (D) the user can go back to the home screen increasing repeatability.

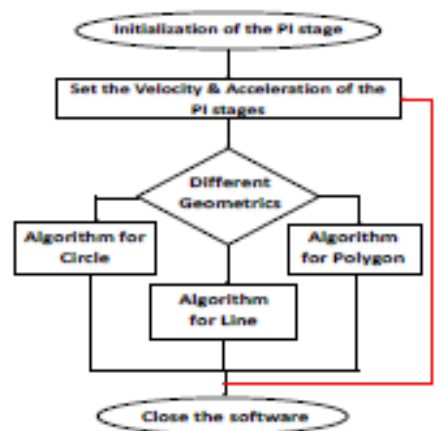

(a) Initial Algorithm

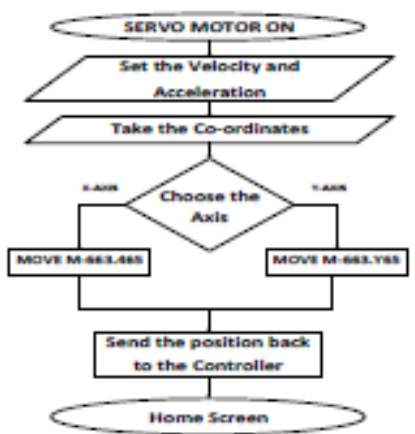

(b) Algorithm for liner Geometries

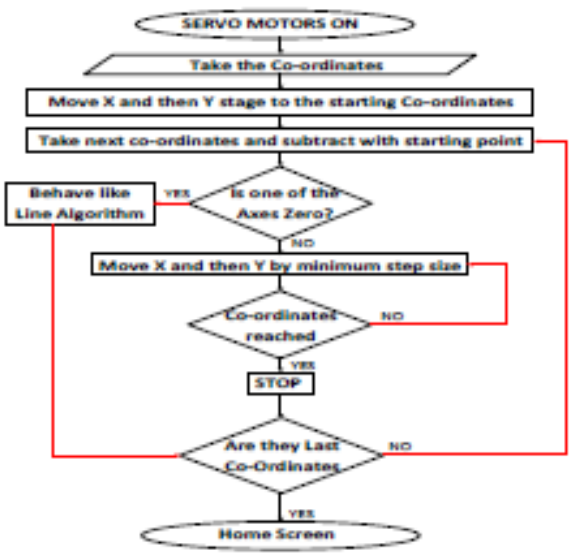

(c) Algorithm for Polygonal Geometry

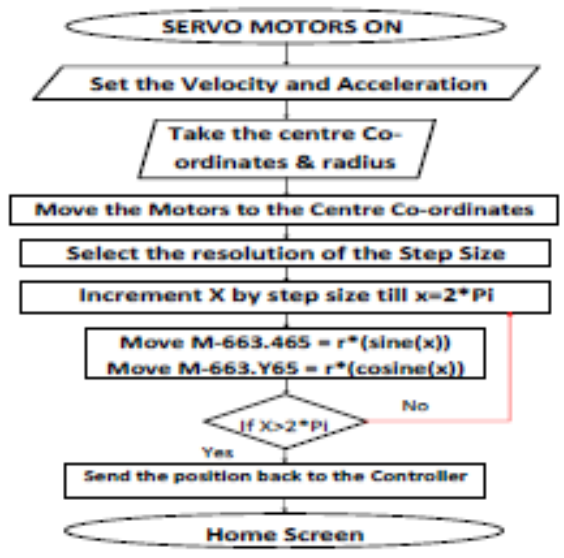

(d) Algorithm for Circular Geometry

Figure 7: Flowcharts for Different Geometries 


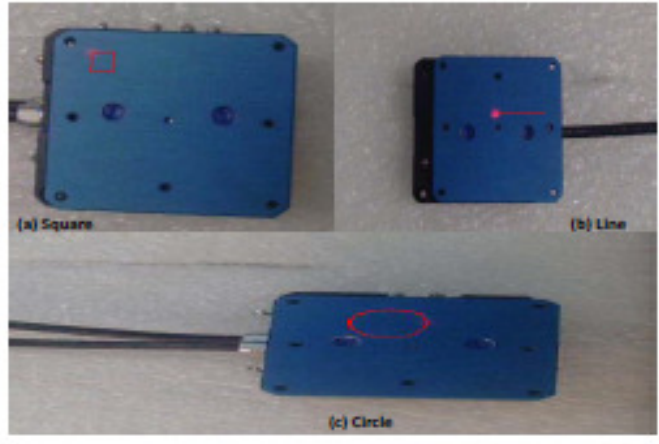

Figure 8: Pattern Made by the Movement of the Stages

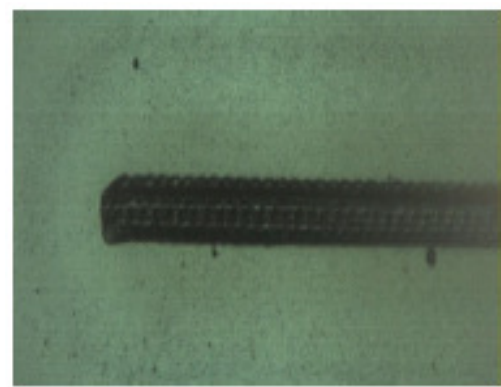

(a)
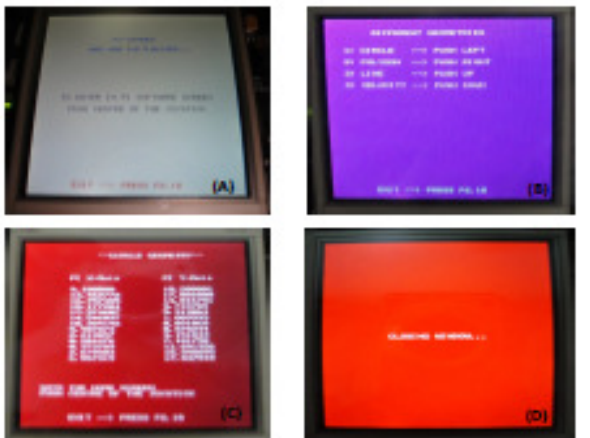

Figure 9: Different Windows of the User Interface

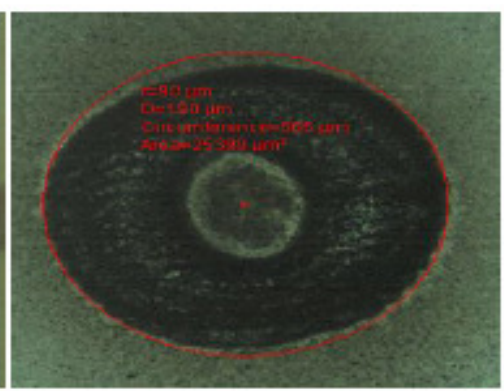

(b)

Figure 10: Manufactured Silicon Workpiece

\section{EXECUTION OF TASKS IN MICRO-MANUFACTURING SYSTEM}

The program was first run on a very raw set up where the 2 stages were not yet placed on top of each other.The movement of the 2 stages was verified to be correct. The stages were then assembled as shown in Fig. 3. The routine was run to verify the joint movement of the stages. Once verified, the set up was placed below the femtosecond laser and the material on which the pattern had to be made was placed on top of the stage, as shown in the Fig.1.The femtosecond laser is acting on the material in short bursts and hence can be co-ordinated well with the movement of the stages. The stages move to expose a different portion of the material to the laser each time which subsequently leads to the creation of the pattern on the materials. Fig. 10 are the photographs of the manufactured material taken inside the light microscope. The line as shown in part (a) was burnt on the material whilst the stages were moving from one extreme end to the other. The part (b) is the circle with a radius of approximately 90 micron.

\section{FUTURE SCOPE}

The kinematics of the micro parts see to it that surface interaction and friction force become more prominent. Also there is a significant problem of spring back characteristics. Hence complex geometries are needed for the tools for increased tolerances and good surface quality [11]. The creation of patterns in three dimensions made by a powerful femtosecond laser pulse, allows us to delve deeper and create further advancements in the fields of fabrication of integrated circuits, Micro electromechanical systems (MEMS), biosensors, Power MEMS and energy harvesters/scavengers[8,13]. Future experiments include plotting and analyzing step 
responses using the optimized P, I, D set of constants, also analyzing the behaviour of the stage under different load conditions. The ARM microcontrollers have a number of integrated peripherals which will help us develop a generalized platform for the user interface.

\section{CONCLUSION}

The software necessary to communicate with 2 controllers was not more than $40 \mathrm{kB}$ in size. The steady state error and the rise time of the time domain response was optimized for a particular experiment. The user interface was self- explanatory and had the option of returning to the home screen after every task. Tracking of the position of the actuator was achieved as shown in Fig. 9 Window (C). The assembly was highly portable. The repeatability was marginally increased along with saving a precious amount of time.

\section{ACKNOWLEDGEMENT}

Authors thank CSIR for funding the research program under Network Project, NWP-30 and CEERI Pilani for creating supporting facilities for the project. Authors also wish to thank Dr. P. Bhanu Prasad, Mr. Talebar Singh and S Mohan Mahalaxmi Naidu \& Amit Patwardhan professors at International Institute of Information Technology, Pune for their valuable discussion and support towards this project.

\section{REFERENCES}

[1] Huixing Zhou, Brian Henson, Andrew Bell, Andrew Blackwood, Andy Beck, Richard Burn "Linear Piezo-Actuator and its Applications".

[2] Michael Gauthier and Emmanuel Piat, "Control of a particular micro-macro positioning system applied to cell micromanipulation", IEEE Transactions on Automation Science and Engineering 3, 3 (2006), pp. 264-271.

[3] Yung-Tien Liu, Y. Yamagata, T. Higuchi, "Micropositioning device using impact force of piezoelectric flying wires", IEEE/ASME Transactions on Mechatronics, Vol. 10, pp.692- 696, Dec. 2005.

[4] Micky Rakotondrabe, Yassine Haddab, and Philippe Lutz, "Development, Modeling, and Control of a Micro /Nanopositioning 2-DOF Stick-Slip Device",IEEE/ASME Transac- tions on Mechatronics, Vol. 14, pp.733-745, Dec. 2009.

[5] "LPC2478-32 Datasheet", NXP Semiconductors, Eindhoven, Netherlands.

[6] "MS178E User Manual C-867 PILine R Controller", Physik Instrumente (PI) GmbH \& Co.KG, Karlsruhe, Germany.

[7] "PI Compact Linear Positioning Stage M-663 Datasheet", Physik Instrumente (PI) GmbH \& Co. KG, Karlsruhe, Germany.

[8] Nadeem H. Rizvi, "Femtosecond laser micromachining: Current status and applications", RIKEN Review No. 50: Focused on Laser Precision Microfabrication (LPM 2002), pp.107-112, January, 2003.

[9] "CPA-Series Ti:Sapphire Ultrashort Pulse Laser-Datasheet" Clark-MXR, Inc, Michigan USA.

[10] Nozomu Mishima, "Development of a Micro Manufacturing System and its Efficiency Evaluation Method" International Conference on Automation Science and Engineering, pp.478 - 483, Shanghai, China, October 7-10, 2006.

[11] Tiesler N, Engel U, "Microforming-effects of miniaturization" In: Pietrzyk M, et al., ed- itors. Proceedings of the International Conference on Metal Forming. Rotterdam, Nether- lands: Balkema; 2000. pp. 355. 
[12] H. Naitoh and S. Tadakuma, "Microprocessor-Based Adjustable-Speed DC Motor Drives Using Model Reference Adaptive Control" IEEE Trans. on Industry Applications, Vol. IA- 23, pp. 313-318, March/April 1987.

[13] Muammer Koc and Tugrul Ozel, "Micro-Manufacturing: Design and Manufacturing of MicroProducts", John Wiley \& Sons, 31-May-2011,pp. 1-24.

[14] Markus G. Bauer,"Design of a Linear High Precision Ultrasonic Piezoelectric Motor",Ph.D.dissertation, North Carolina State University , Dept. of Mechanical Engineering, Raleigh, 2001.

[15] O. Vyshnevskyy, S. Kovanev, W. Wischnewskiy, J. Mehner,” New Type of Piezoelectric Standing Wave Ultrasonic Motors with Cylindrical Actuators", The 9th International Con- ference on New Actuators and the 3rd International Exhibition on Smart Actuators and Drive Systems, Bremen, Germany.

[16] Z.Tao and B.Bhushan,"New Techniques for studying nanoscale friction at sliding velocities up to $200 \mathrm{~mm} . \mathrm{s}$ using atomic force microscope" REVIEW of SCIENTIFIC INSTRUMENTS 77,103705(2006). 